\title{
Medical treatment of ascites in cirrhosis
}

\author{
Alexander L. Gerbes \\ Department of Medicine II, Klinikum Grosshadern, University of Munich, Germany
}

\begin{abstract}
Medical treatment of cirrhotic ascites is essentially supportive, dictated by the patient's discomfort, impaired cardiovascular or respiratory function and potential for infection. Treatment of 'simple' ascites (moderate fluid accumulation, serum albumin $>3.5 \mathrm{~g} / \mathrm{dl}$, serum creatinine $<1.5 \mathrm{mg} / \mathrm{dl}$, no electrolyte disturbance) is implemented sequentially. Only $10 \%$ of patients respond to dietary sodium restriction and bed rest; most require pharmacotherapy consisting of spironolactone, which increases the proportion of responding patients to $65 \%$ and loop diuretics, which may produce clinical improvement in an additional $20 \%$ ( $85 \%$ in all); in the remaining $15 \%$ of refractory patients, use of novel adjunctive therapies may be attempted. Patients with tense ascites, impaired renal function and electrolyte disturbances merit special consideration before diuretics are introduced. Spironolactone has long been a standard for the treatment of cirrhotic ascites because it directly antagonizes aldosterone. The loop diuretic most frequently added to spironolactone has been furosemide. However, there is preliminary evidence that torasemide may be more effective in some patients. Other investigational agents that may play a role in treatment of patients resistant to conventional drugs include ornipressin (a vasopressin analogue) and atrial natriuretic factor.
\end{abstract}

Key words: Ascites; Atrial natriuretic peptide; Cirrhosis; Diuretics; Liver disease; Ornipressin; Spironolactone; Torasemide

A brief overview of the pathophysiology of ascites formation in hepatic cirrhosis (1) can facilitate understanding both of the available therapeutic options and of the rationale for development of new therapeutic approaches. Traditionally, the initiating event of renal sodium and water retention in cirrhosis was considered to be ascites formation (underfilling hypothesis) or primary renal dysfunction due to a hepatorenal reflex (overflow hypothesis) (2). The alterations of systemic, splanchnic and renal haemodynamics, as well as increases in circulating levels of substances that cause sodium retention (3-6), are compatible with a decrease in effective blood volume as suggested by the underfilling hypothesis. These alterations, however, precede ascites formation. The recently introduced vasodilation hypothesis (7) reconciles many aspects of the underfill- ing theory and the overflow theory; it proposes that peripheral arterial vasodilation is the initiating event leading to decreased effective blood volume and renal sodium retention (Table 1) and suggests that haemodynamic, hormonal and renal changes are augmented as liver disease becomes more severe. Peripheral arterial vasodilation leads to a decrease in effective arterial blood volume and, in compensation, increases in circulating levels of renin, aldosterone, noradrenaline and vasopressin, which result in renal vasoconstriction with sodium and water retention. With increasing severity of cirrhosis, the activation of these stimulants of sodium retention cannot restore effective blood volume. This, together with a decrease in plasma oncotic pressure due to hypoalbuminaemia and an increase in hydrostatic pressure in the splanchnic vessels, results in extravasation of fluid. Once the resorptive

Correspondence to: Priv. Doz. Dr. Alexander L. Gerbes, Medizinische Klinik II, Klinikum Grosshadern, Universität München, Marchioninistraße 15, W 8000 München 70, Germany. 


\section{TABLE}

Vasodilation hypothesis of ascites formation: Sequence of events

1. Cirrhosis

2. Peripheral arterial vasodilation

3. Decrease in effective blood volume

4. Activation of volume-retaining hormones

5. Renal sodium retention

6. Increase in blood volume

7. Ascites

capacity of the lymphatic vessels is exceeded, ascites becomes overt.

After the diagnosis of ascites is established by clinical examination and ultrasonography, the cause of the condition must be determined. The patient's medical history and results of typical laboratory assays can often reveal malignant causes (e.g. peritoneal carcinomatosis and liver metastasis) or non-malignant causes (e.g. cirrhosis of the liver). Laboratory examination of the ascitic fluid can be helpful in differentiating the cause of ascites (8-10), particularly determinations of cholesterol, carcinoembryonic antigen and other tumour markers in addition to cell count and differentiation; bacteriologic and cytologic examinations also play an important role in differential diagnosis (Fig. 1).

Ascites does not require treatment merely because it exists. However, most patients with ascites feel uncomfortable and their physical activity is limited. Furthermore, therapy for ascites is indicated by the impairment of cardiovascular and respiratory functions due to tense ascites and the potential development of spontaneous bacterial peritonitis. Several therapeutic options are available for the management of ascites in patients with hepatic cirrhosis: bed rest, restriction of sodium intake, diuretics, paracentesis, temporary or continuous reinfusion procedures (such as peritoneovenous shunting), portacaval or intrahepatic shunting procedures and liver

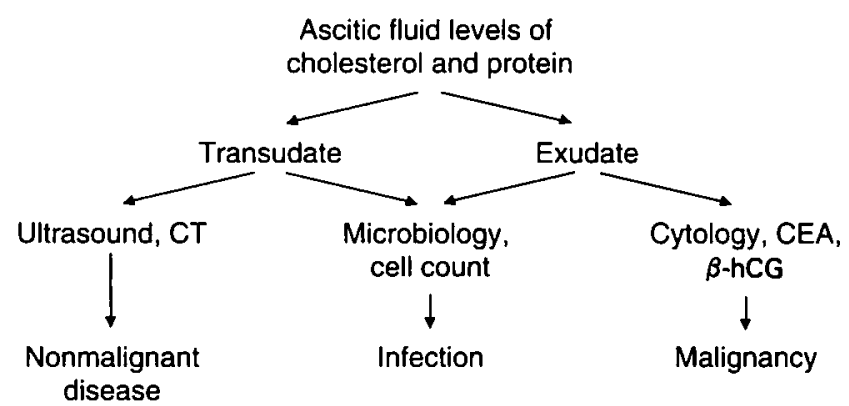

Fig. 1. Laboratory analyses of ascitic fluid and imaging techniques valuable in the differential diagnosis of ascites. $\beta$-hCG, $\beta$-human chorionic gonadotropin; CEA, carcinoembryonic antigen; $C T$, computed tomography. transplantation. This article concentrates on medical therapy for ascites which, like all procedures other than transplantation, is only supportive. Therefore, the principle of 'primum nil nocere' should always be kept in mind (11-13).

\section{Medical therapeutic options}

Sodium balance can be achieved more easily when sodium intake is restricted. Limitation to about $50 \mathrm{mmol}$ of sodium, which corresponds to approximately $3 \mathrm{~g}$ of dietary salt, is usually well tolerated. Lower levels of sodium intake result in less palatable diets and thus lead to poorer patient compliance; severely restricted sodium intake may also contribute to increased activation of the renin-angiotensin-aldosterone system. Bed rest alone has a diuretic effect that can augment the natriuresis induced by diuretic drugs (14-16).

The results of randomised, controlled trials have disclosed the following about diuretic therapy:

- Sodium excretion is induced by combination therapy with spironolactone and furosemide (17).

- In the setting of cirrhosis, spironolactone alone or combined with furosemide induces greater sodium excretion than furosemide alone $(18,19)$.

- Restriction of salt and water intake alone is less efficient than salt and water restriction plus the use of spironolactone or the combination of spironolactone and furosemide (20).

Based on these findings, sequential therapy for ascites is usually recommended (Fig. 2).

\section{Sequential approach to ascites management}

Sodium restriction and bed rest are sufficient to manage ascites in about $10 \%$ of patients. However, because an adequate response (mean reduction in body wt. $\approx 400 \mathrm{~g} /$ day for 4 days) will not be seen in most patients, administration of spironolactone will be required. Ascites can be alleviated in approximately $65 \%$ of patients with spironolactone, the mainstay of diuretic therapy for ascites, at dosages of up to $300 \mathrm{mg} /$ day orally. Addition of a loop diuretic (e.g. furosemide) at dosages of up to $120 \mathrm{mg} /$ day orally increases the rate of therapeutic success to approximately $85 \%$. In about $15 \%$ of patients, ascites remains refractory to diuretic therapy or treatment must be discontinued because of side 


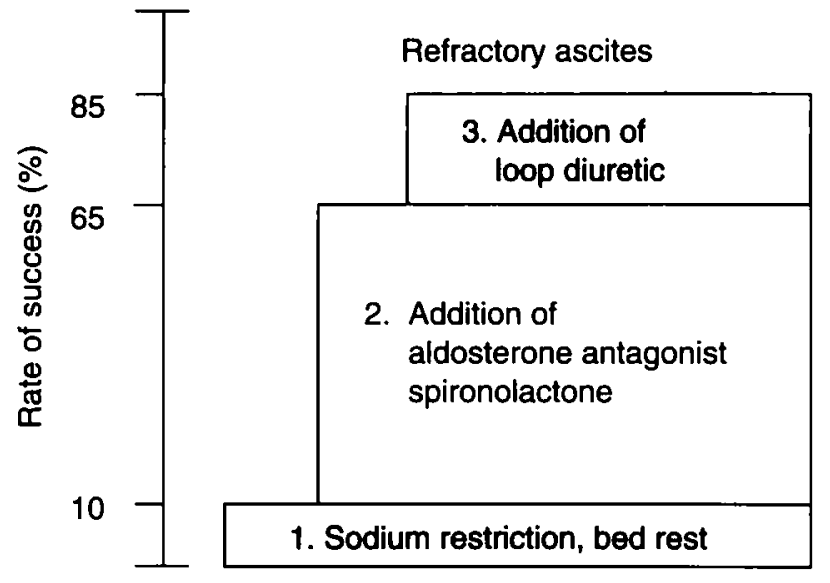

Fig. 2. Sequential approach to ascites management in hepatic cirrhosis and rate of therapeutic success.

effects, such as encephalopathy, hyponatraemia, hypokalaemia, or hyperkaemia.

\section{Guidelines for optimum benefit}

The patient with ascites should be thoroughly evaluated after treatment with a balanced sodium diet alone for at least 5 days. This clinical evaluation must include measurement of blood pressure and heart rate as well as examinations for encephalopathy and muscular and neurologic abnormalities. At the same time, serum should be analysed for concentrations of sodium, potassium, creatinine, urea, calcium, magnesium, albumin and uric acid and serum osmolality should be assessed. Recommendations also include weighing the patient daily and analysing 24-h urine collections every other day. Urinalysis should measure concentrations of sodium, potassium and creatinine, as well as urinary osmolality, which will allow for the calculation of creatinine clearance, fractional sodium excretion and the urinary sodium/potassium ratio. In addition, laboratory examination of the urinary sediment may indicate glomerular or tubular damage. After this initial evaluation, the patient may be classified as having 'simple' or 'complex' ascites.

\section{Managing simple ascites}

Patients may be regarded as having simple ascites when there is no evidence of serum electrolyte abnormalities, hypoalbuminaemia, or encephalopathy; no major reduction in glomerular filtration rate; no outstanding sodium retention; and no more than a moderate accumulation of ascitic fluid (Table 2). The condition of these patients will likely respond to the con-
TABLE 2

Characteristics of 'simple' ascites suggesting sequential diuretic therapy

Moderate degree of ascites

No encephalopathy

Urinary sodium excretion $>20 \mathrm{mmol} /$ day

Fractional sodium excretion $>0.3 \%$

Levels of serum constituents

Sodium $>130 \mathrm{mmol} / \mathrm{l}$

Potassium 3.6-4.9 mmol/

Albumin $>3.5 \mathrm{~g} / \mathrm{dl}$

Creatinine $<1.5 \mathrm{mg} / \mathrm{dl}$

ventional sequential therapy of ascites. Spironolactone is the initial agent of choice rather than a loop diuretic because it directly antagonizes aldosterone, the pivotal hormone in oedematous disorders. Moreover, spironolactone treatment generally avoids the overly brisk diuresis, natriuresis and potassium loss associated with loop diuretics.

If simple ascites does not respond adequately to diuretic therapy, several possibilities should be considered. A lack of decrease in body weight, despite marked urinary sodium excretion, indicates a high sodium intake; this may be due to dietary noncompliance or to inadvertent iatrogenic sodium administration (e.g. antibiotics, antacids). Because amino acids increase glomerular filtration rate, a diet severely restricted in protein (e.g. to prevent encephalopathy) may impair renal function. In addition, renal function can be affected by use of cyclooxygenase inhibitors, typified by non-steroidal anti-inflammatory drugs such as indomethacin; by volume depletion resulting from lactulose-induced diarrhoea; and by overly vigorous diuresis. Furthermore, some drugs (e.g. aminoglycosides) may directly damage the renal tubule. In the absence of the preceding possibilities, a lack of response to diuretic treatment might indicate fulminant hepatitis, spontaneous bacterial peritonitis or gastrointestinal bleeding.

\section{Managing complex ascites}

Whether complex ascites exists initially or develops during therapy, its management needs special attention. Hyponatraemia is largely caused by dilution and, therefore, should be managed with fluid restriction. Hypokalaemia demands potassium replacement or a modification of the diuretic regimen; the latter is also indicated in hyperkalaemia. Patients whose serum albumin concentration is $<3.5 \mathrm{~g} / \mathrm{dl}$ may benefit from the intravenous administration of salt-poor albumin (21), although the value of this intervention has not been clearly demonstrated by clinical studies. Spontaneous bacterial peritonitis must be managed with antibiotics. 
Severe hepatic encephalopathy requires treatment (e.g. by stringent dietary protein limitation, lactulose administration and, possibly, consideration of liver transplantation.

Tense ascites may be relieved initially by therapeutic paracentesis (22); an adequate diuretic dosage can then be established (23). Diuretic therapy should not routinely be started in patients whose serum creatinine concentrations are $>2 \mathrm{mg} / \mathrm{dl}$ because of the risk of azotaemia and renal failure.

\section{Minimizing side sects}

Side effects of therapy for ascites may be minimized by monitoring urinary volume and electrolyte excretion and maintaining normal levels of serum electrolytes and renal function. Side effects may also be minimized by controlling body weight, blood pressure and heart rate. To avoid reducing the effective blood volume and further activating mechanisms of sodium retention in patients without peripheral oedema, diuretic dosage should be adjusted to provide a daily weight loss of no more than 750-900 g. However, patients with peripheral oedema can tolerate a daily weight loss of $\geq 1.5 \mathrm{~kg}$ without significant side effects $(23,24)$.

\section{Investigational agents for use in refractory ascites}

In the minority of patients who fail to respond to conventional therapies, which are based on bed rest, sodium restriction and administration of spironolactone (up to a maximum of $300 \mathrm{mg} / \mathrm{day}$ ) and the subsequent addition of furosemide (up to a maximum of 120 $\mathrm{mg} /$ day), three other agents currently under investigation may be tried. One of them, torasemide, is a loop diuretic, while the other two, atrial natriuretic peptide (ANP) and ornipressin, act as hormones.

\section{Torasemide}

A now loop diuretic, torasemide, has a bioavailability of $>85 \%(25)$. Compared with furosemide, torasemide has a longer half-life and smoother, more prolonged activity; these features may be advantageous for cirrhotic patients, who are sensitive to sudden decreases in intravascular volume.

A recent study found that in patients with ascites, torasemide induced a prolonged natriuresis and stronger diuresis than furosemide (26). Of particular interest, patients whose ascites did not respond to administration of furosemide or to a combination of spironolactone and furosemide exhibited significantly greater natriuresis after receiving torasemide or the combination of spironolactone and torasemide, respectively $(26,27)$. However, further investigation is needed to establish torasemide as the preferred loop diuretic in cirrhotic patients with ascites.

\section{Atrial natriuretic factor ( $A N F$ )}

The potential therapeutic application of ANF (or its analogues) to patients with ascites has recently received attention $(28,29)$ despite the fact that ANF must be administered intravenously and has a short duration of action. It increases glomerular filtration rate, influences tubuloglomerular feedback and decreases the release of renin; it also inhibits the synthesis and release of aldosterone (30). However, in animal models and patients with cirrhosis, particularly those with ascites, the renal response to ANF seems to be blunted $(31,32)$. This might be due to alterations in renal ANF receptors (33) as well as to a decrease in mean arterial pressure following ANF administration in cirrhotic subjects (34). Thus, low-dose infusions of ANF may induce greater natriuresis than high-dose infusions.

Coadministration of vasoconstrictors might prevent ANF-induced hypotension. In an animal model of portal hypertension, combination with vasopressin was shown to potentiate the natriuresis and diuresis induced by ANF $(35,36)$. Clinical evaluation of such combination therapy has not yet been pursued. The development of inhibitors of the ANF-degrading proteinase EC 3.4.24.11 or of ANF analogues that selectively bind to clearance receptors (37) may result in a longer-acting oral agent, which could prompt clinical studies.

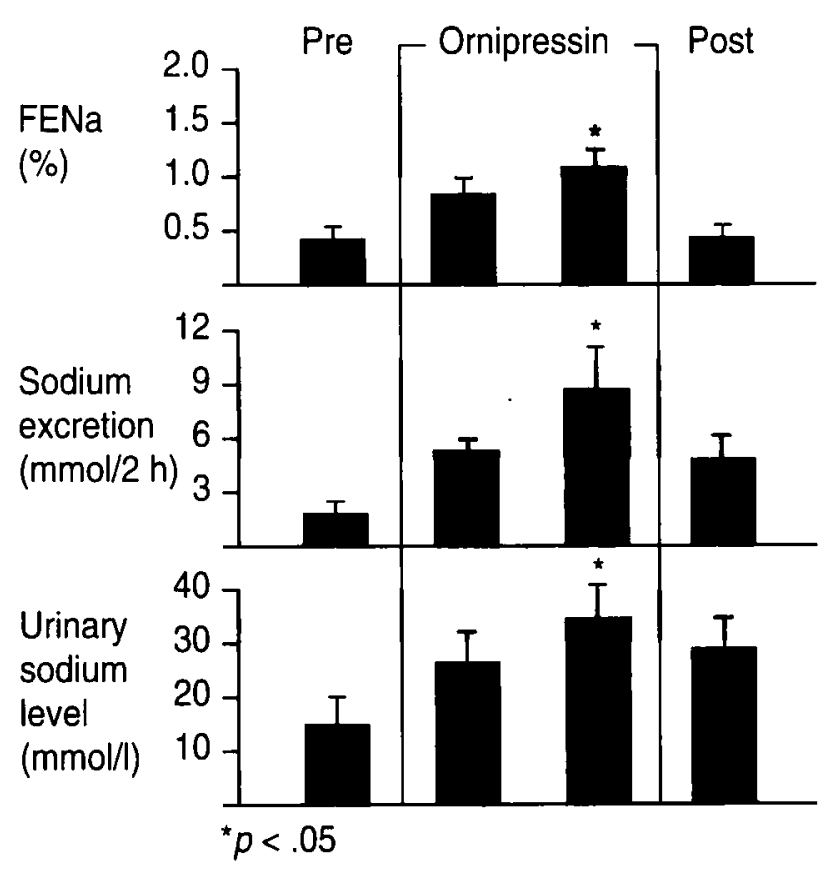

Fig. 3. Changes in renal sodium excretion after 2- and 4-h infusions of ornipressin and $2 \mathrm{~h}$ after infusion termination (Lenz et al., 1991). Shading indicates normal ranges. FENa, fractional elimination of sodium. 


\section{Ornipressin}

The effects of the vasopressin analogue ornipressin were recently studied in patients with poor renal function who were treated in an intensive care unit (38). After 2-h and 4-h infusions, peripheral vasodilation and the renal vasoconstriction reversed in part and were accompanied by a decrease in activation of the mechanisms of sodium retention and an increase in circulating levels of ANF. These effects resulted in improved renal function and sodium excretion (Fig. 3). Ornipressin infusion thus may be helpful as a short-term intervention to improve the critical condition of such patients.

\section{Additional alternatives}

Other therapeutic approaches may prove useful, particularly in patients whose ascites is resistant to conventional diuretic therapy. The development of antagonists to block the increased synthesis of leukotrienes $(39,40)$ and to attenuate the effects of platelet-activating factor $(41,42)$ might be helpful, particularly for patients with renal impairment.

\section{Acknowledgements}

This work has been supported by the Deutsche Forschungsgemeinschaft (Ge 576/2-2). The author wishes to thank F. Ringel and J. Nagl for their assistance in preparing the manuscript.

\section{References}

1 Gerbes AL. Pathophysiology of ascites formation in cirrhosis of the liver. Hepatogastroenterology 1991; 38: 360-4.

2 Levy $M$. Sodium retention and ascites formation in dogs with experimental portal cirrhosis. Am J Physiol 1977; 233: F572-85.

3 Bichet DG, Van Putten VJ, Schrier RW. Potential role of increased sympathetic activity in impaired sodium and water excretion in cirrhosis. N Engl J Med 1982; 307: 1552-7.

4 Bosch J, Arroyo V, Betriu A, et al. Hepatic hemodynamics and the renin-angiotensin-aldosterone system in cirrhosis. Gastroenterology 1980; 78: 92-9.

5 DiBona GF. The functions of renal nerves. Rev Physiol Biochem Pharmacol 1982; 94: 75-181.

6 Henriksen JH, Ring-Larsen H, Christensen NJ. Circulating noradrenaline and central haemodynamics in patients with cirrhosis. Scand J Gastroenterol 1985; 20: 1185-90.

7 Schrier RW, Arroyo V, Bernardi M, Epstein M, Henriksen JH, Rodés J. Peripheral arterial vasodilation hypothesis: a proposal for the initiation of renal sodium and water retention in cirrhosis. Hepatology 1988; 8: 1151-7.

8 Jüngst D, Gerbes AL, Martin R, Paumgartner G. Value of ascitic lipids in the differentiation between cirrhotic and malignant ascites. Hepatology 1986; 6: 239-43.

9 Gerbes AL, Jüngst D, Xie Y, Permanetter W, Paumgartner G. Ascitic fluid analysis for the differentiation of malignancyrelated and nonmalignant ascites: proposal of a diagnostic sequence. Cancer 1991; 68: 1808-14.

10 Hoermann R, Gerbes AL, Spoettl G, Juengst D, Mann K. Immunoreactive human chorionic gonadotropin and its free beta- subunit in serum and ascites of patients with malignant tumors. Cancer Res 1992; 52: 1520-4.

11 Conn HO. Diuresis of ascites: fraught with or free from hazard. Gastroenterology 1977; 73: 619-21.

12 Sherlock S, Shaldon S. The aetiology and management of ascites in patients with hepatic cirrhosis: a review. Gut 1963; 4: 95-105.

13 Schölmerich J. Aszites. 1st ed. Berlin, Heidelberg: Springer Verlag, 1991.

14 Karnad DR, Tembulkar P, Abraham P, Desai NK. Head-down tilt as a physiological diuretic in normal controls and in patients with fluid-retaining states. Lancet 1987 ; ii: $525-8$.

15 Bernardi M, Santini C, Trevisani F, Baraldini M, Ligabue A, Gasbarrini $G$. Renal function impairment induced by change in posture in patients with cirrhosis and ascites. Gut 1985; 26 : 629-35.

16 Ring-Larsen H, Henriksen JH, Wilken C, Clausen J, Pals H, Christensen NJ. Diuretic treatment in decompensated cirrhosis and congestive heart failure: effect of posture. $\mathrm{Br}$ Med $\mathrm{J}$ 1986; 292: 1351-3.

17 Fuller RK, Khambatta PB, Gobezie GC. An optimal diuretic regimen for cirrhotic ascites. A controlled trial evaluating safety and efficacy of spironolactone and furosemide. J Am Med Assoc 1977; 237: 972-5.

18 Fogel MR, Sawhney VK, Neal EA, Miller RG, Knauer CM, Gregory PB. Diuresis in the ascitic patient: a randomized controlled trial of three regimens. J Clin Gastroenterol 1981; 3 (Suppl. 1): 73-80.

19 Perez-Ayuso RM, Arroyo V, Planas R, et al. Randomized comparative study of efficacy of furosemide versus spironolactone in nonazotemic cirrhosis with ascites. Gastroenterology 1983; 84: 961-8.

20 Gregory PB, Broekelschen PH, Hill MD, et al. Complications of diuresis in the alcoholic patient with ascites: a controlled trial. Gastroenterology 1977; 73: 534-8.

21 Faloon WW, Eckhardt RD, Murphy TL, Cooper AM, Davidson CS. An evaluation of human serum albumin in the treatment of cirrhosis of the liver. J Clin Invest 1949; 28: 583-94.

22 Ginès P, Arroyo V, Quintero E, et al. Comparison of paracentesis and diuretics in the treatment of cirrhotics with tense ascites. Results of a randomized study. Gastroenterology 1987; 93: 234-41.

23 Shear L, Ching S, Gabuzda GJ. Compartmentalization of ascites and edema in patients with hepatic cirrhosis. N Engl J Med 1970; 282: 1391-6.

24 Pockros PJ, Reynolds TB. Rapid diuresis in patients with ascites from chronic liver disease: the importance of peripheral edema. Gastroenterology 1986; 90: 1827-33.

25 Broekhuysen J, Deger F, Douchamps J, Ducarne H, Herchuelz A. Torasemide, a new potent diuretic. Double-blind comparison with furosemide. Eur J Pharmacol 1986; 31 (Suppl.): 29-34.

26 Gerbes AL, Bertheau-Reith U, Falkner C, Jüngst D, Paumgartner $\mathrm{G}$. Advantages of the new loop diuretic torasemide over furosemide in patients with cirrhosis and ascites. A randomized double-blind cross-over study. J Hepatol 1992: in press.

27 Laffi G, Marra F, Buzzelli G, et al. Comparison of the effects of torasemide and furosemide in nonazotemic cirrhotic patients with ascites: a randomized, double-blind study. Hepatology 1991; 13: 1101-5.

28 Gerbes AL, Arendt RM, Paumgartner G. Editorial. Atrial natriuretic factor. Possible implications in liver disease. J Hepatol 1987; 5: 123-32.

29 Epstein M. Atrial natriuretic factor in patients with liver disease. Am J Nephrol 1989; 9: 89-100.

30 Gerbes AL, Arendt RM, Gerzer R, et al. Role of atrial natriuretic factor, cyclic GMP and the renin-aldosterone system in acute volume regulation of healthy human subjects. Eur J Clin Invest 1988; 18: 425-9.

31 Gerbes AL, Wernze H, Arendt RM, Riedel A, Sauerbruch T, Paumgartner G. Atrial natriuretic factor and renin-aldosterone in volume regulation of patients with cirrhosis. Hepatology 1989; 9: 417-22.

32 Gerbes AL, Arendt RM, Stangl E, Gülberg V, Sauerbruch T, Jüngst $D$, Paumgartner $G$. Characterization of the ANF system in patients with cirrhosis of the liver. In: Forssmann WG, 
Scheuermann DW, Alt J, eds. Functional Morphology of the Endocrine Heart. Steinkopff, Darmstadt, 1989; 229-34.

33 Gerbes AL, Kollenda MC, Volimar AM, Reichen J, Vakil N, Scarborough RM. Altered density of glomerular binding sites for atrial natriuretic factor in bile duct-ligated rats with ascites. Hepatology 1991; 13: 562-6.

34 Petrillo A, Scherrer U, Gonvers JJ, et al. Atrial natriuretic peptide administered as intravenous infusion or bolus injection to patients with liver cirrhosis and ascites. J Cardiovasc Pharmacol 1988; 12: 279-85.

35 Gerbes AL. Augmentation of the natriuretic response to atrial natriuretic factor in cirrhosis. (Letter). Hepatology 1989; 10: 897-8.

36 Ganger DR, Gottstein J, Blei AT. Hemodynamic and renal effects of atrial natriuretic factor in portal hypertensive rats. Potentiation by Phe-Ile-Orn-vasopressin. J Pharmacol Exp Ther 1988; 246: $941-5$.

37 Gerbes AL, Vollmar AM. Degradation and clearance of atrial natriuretic factors (ANF). Life Sci 1990; 47: 1173-80.
38 Lenz K, Hörtnagl H, Druml W, et al. Ornipressin in the treatment of functional renal failure in decompensated liver cirrhosis: effects on renal hemodynamics and atrial natriuretic factor. Gastroenterology 1991; 101: 1060-7.

39 Moore KP, Taylor GW, Maltby NH, et al. Increased production of cysteinyl leukotrienes in hepatorenal syndrome. J Hepatol 1990; 11: 263-71.

40 Keppler D, Huber M, Baumert T. Leukotrienes as mediators in diseases of the liver. Semin Liver Dis 1988; 8: 357-66.

41 Guarner F, Wallace JL, MacNaughton WK, Ibbotson GC, Arroyo V, Rodés J. Endotoxin-induced ascites formation in the rat: partial mediation by platelet-activating factor. Hepatology 1989; 10: 788-94.

42 Caramelo C, Fernandez-Gallardo S, Santos JC, et al. Increased levels of platelet-activating factor in blood from patients with cirrhosis of the liver. Eur J Clin Invest 1987; 17: 7-11. 